\title{
Efficacy of electroacupuncture combined with probiotics for depression and chronic diarrhea in patients and effect on serum inflammatory cytokines, $\mathrm{NE}$ and BDNF
}

\author{
WEI HUANG ${ }^{1 *}$, WEI GAN ${ }^{1 *}$, AN HUANG $^{2}$, YULEI FU ${ }^{3}$, YUZHI SHANG $^{3}$, YUE CHEN $^{3}$, \\ ZHAO TIAN $^{4}$, YUN ZHANG ${ }^{5}$ and GANG FANG ${ }^{5}$ \\ ${ }^{1}$ The First Clinical Faculty, Guangxi University of Traditional Chinese Medicine, Nanning, Guangxi 530023; \\ ${ }^{2}$ College of Pharmacy, Minzu University of China, Beijing 100081; ${ }^{3}$ Graduate School, Guangxi University of \\ Chinese Medicine, Nanning, Guangxi 530001; ${ }^{4}$ Graduate School of Hubei University for Nationalities, \\ Enshi, Hubei 445000; ${ }^{5}$ Laboratory of Zhuang Medicine Prescriptions Basis and Application Research, \\ Guangxi University of Chinese Medicine, Nanning, Guangxi 530001, P.R. China
}

Received January 21, 2019; Accepted May 15, 2019

DOI: $10.3892 / \mathrm{etm} .2019 .7977$

\begin{abstract}
Efficacy of electroacupuncture combined with probiotics for depression and chronic diarrhea in patients, and its effect on the levels of serum inflammatory cytokines, norepinephrine (NE) and brain-derived neurotrophic factor (BDNF) were investigated. A total of 104 patients with depression and chronic diarrhea admitted to The First Clinical Faculty, Guangxi University of Traditional Chinese Medicine from July 2014 to June 2018 were randomly divided into the observation group $(n=56)$ and the control group $(n=48)$. The observation group was treated with electroacupuncture combined with probiotics, and the control group was given conventional drugs for depression and chronic diarrhea. The Hamilton Depression Rating Scale (HAM-D) score and the abdominal symptom score were evaluated before treatment and at 3 weeks after treatment. Changes in the levels of serum inflammatory cytokines [interleukin (IL)-6, IL-2 and tumor necrosis factor (TNF)- $\alpha$ ] as well as the levels of NE and BDNF in the two groups of patients before and after treatment were determined using radioimmunoassay. Compared with those in the control group, the symptoms of depression and diarrhea in the observation group were remarkably alleviated $(\mathrm{p}<0.05)$. After treatment, the serum cytokine levels in the two groups
\end{abstract}

Correspondence to: Dr Yun Zhang or Dr Gang Fang, Laboratory of Zhuang Medicine Prescriptions Basis and Application Research, Guangxi University of Chinese Medicine, 179 Mingxiu East Road, Nanning, Guangxi 530001, P.R. China

E-mail: zuch336@163.com

E-mail:000573@gxtcmu.edu.cn

*Contributed equally

Key words: electroacupuncture, probiotics, depression, chronic diarrhea, cytokine, norepinephrine, brain-derived neurotrophic factor of patients were decreased, and the decreased level of serum inflammatory cytokines in the observation group was not obviously different from that in the control group. Besides, the serum BDNF level in the observation group was also reduced $(p<0.05)$. The overall efficacy of the observation group was superior to that of the control group, showing a statistical difference. Electroacupuncture combined with probiotics brings good efficacy to patients with depression and chronic diarrhea, which is worthy of clinical promotion and development.

\section{Introduction}

As a disease of the chronic digestive system, chronic diarrhea is commonly manifested as changes in bowel habits, diarrhea and abdominal pain as well as abdominal distension in a few cases. The pathogenesis of the disease is mostly the intestinal flora imbalance and ectopia, endocrine dysfunction and mental stress. Besides, excessive mental stress often causes neuroendocrine dysfunction, with such manifestations as the secretion disorders of corticotropin-releasing hormone and adrenocorticotropic hormone (1), which lead to mental mania or apathy, delusion and depression in patients and induce the onset of depression in patients.

Increasingly more studies have revealed that inflammatory cytokines also exert crucial effects in the onset process of depression. Both in adequate and excessive secretions of collective anti-inflammatory cytokines are the most important mechanisms of chronic diarrhea. Excessively secreted inflammatory cytokines can induce depression, thus resulting in a variety of symptoms of depression such as sexual hypoactivity, reduced social activity, slow action and sleep disorders (2), and studies have manifested that the changes in the level of cytokines have a correlation with the occurrence of depression in patients (3). The present study aimed to observe the efficacy of electroacupuncture combined with probiotics in the treatment of patients with depression and chronic diarrhea and its influence on cytokines, and to investigate the correlations of cytokines with the occurrence and prognosis of the disease. 
Table I. Comparison of the HAM-D score between the two groups of patients (mean \pm SD).

\begin{tabular}{lccrc}
\hline & \multicolumn{2}{c}{ Observation group $(\mathrm{n}=56)$} & \multicolumn{2}{c}{ Control group (n=48) } \\
\cline { 2 - 3 } Items & Before treatment & After treatment & & Before treatment \\
\hline Total score of HAM-D & $32.34 \pm 5.43$ & $15.57 \pm 3.79$ & $29.34 \pm 6.09$ & $14.34 \pm 4.65$ \\
Weight & $2.47 \pm 1.08$ & $0.89 \pm 0.47$ & $1.97 \pm 2.16$ & $1.03 \pm 0.66$ \\
Sleep disorder & $5.42 \pm 2.13$ & $1.45 \pm 1.34$ & $5.86 \pm 3.18$ & $2.95 \pm 2.13^{\mathrm{a}}$ \\
Anxiety/somatization & $6.36 \pm 4.24$ & $4.21 \pm 1.93$ & $5.96 \pm 4.67$ & $3.84 \pm 1.82$ \\
Day and night change & $1.72 \pm 0.86$ & $0.85 \pm 0.47$ & $1.57 \pm 0.67$ & $0.99 \pm 0.83$ \\
Retardant & $7.48 \pm 3.54$ & $4.16 \pm 2.35$ & $6.95 \pm 2.64$ & $4.67 \pm 3.12$ \\
Feeling of despair & $5.36 \pm 2.37$ & $3.58 \pm 1.83$ & $4.97 \pm 1.86$ & $3.74 \pm 2.11$ \\
Cognitive disorder & $6.84 \pm 3.83$ & $3.68 \pm 2.85$ & $7.46 \pm 2.93$ & $5.17 \pm 2.04^{\mathrm{a}}$ \\
\hline
\end{tabular}

${ }^{\mathrm{a}} \mathrm{P}<0.05$ vs. control group after treatment. HAM-D, Hamilton Depression Rating Scale; SD, standard deviation.

\section{Patients and methods}

Study subjects. A total of 104 patients with depression and chronic diarrhea admitted to The First Clinical Faculty, Guangxi University of Traditional Chinese Medicine (Nanning, China) from July 2014 to June 2018 were selected, and they were divided into the observation group $(n=56)$ and the control group $(n=48)$. The diagnostic criteria for depression were based on the 10th revision of the International Statistical Classification of Diseases and Related Health Problems (4) and the 3rd revision of the Chinese Classification of Mental Disorders. There were no statistically significant differences in age, sex, course of the disease and complication with other underlying diseases between the two groups of patients $(\mathrm{p}>0.05)$.

The present study was approved by the Ethics Committee of The First Clinical Faculty, Guangxi University of Traditional Chinese Medicine. The signed informed consents were obtained from the patients or the guardians.

Treatment methods. Patients in the control group took orally trimebutine maleate tablets (100 mg/time, 3 times/day) and meptintin (100 mg/day, and the dose was adjusted according to the patient's condition and side effects). Patients in the observation group were treated with electroacupuncture and oral probiotics, $50 \mathrm{~mm}$ acupuncture needles were used to acupuncture such acupoints as Baihui point, Sanyinjiao point and Yanglingquan point. After the air was obtained, the electroacupuncture machine was connected for electroacupuncture for $30 \mathrm{~min}$ each day. Additionally, the patients took viable bifidobacterium quadruple tablets $(1.5 \mathrm{~g} / \mathrm{time}$, 3 times/day) with warm water after meals. Blood was drawn from the two groups of patients for scale evaluation before and after treatment.

Scale assessment. The two groups of patients were scored by Hamilton Depression Rating Scale (HAM-D) before and after treatment. Criteria for chronic diarrhea: diarrhea $\geq 3$ times/day, thin or watery stool and no obvious pus and blood accompanied with abdominal pain, abdominal distension and tenesmus and duration $>3$ weeks (5).
Detection methods. The venous blood of patients was taken at admission and at 3 weeks after treatment. After centrifugation at $6,000 \mathrm{xg}$, for $10 \mathrm{~min}$ at $4^{\circ} \mathrm{C}$, the supernatant was taken, and the changes in the levels of serum inflammatory cytokines interleukins (ILs) and the tumor necrosis factor (TNF)- $\alpha$ ), norepinephrine (NE) and brain-derived neurotrophic factor (BDNF) were detected by means of radioimmunoassay. Fecal flora culture: Approximately $5 \mathrm{~g}$ sterile feces of the patients were taken, diluted with normal saline, shaken, homogenized, inoculated on the selective medium and cultured in an incubator at $35^{\circ} \mathrm{C}$ for $24 \mathrm{~h}$. Finally, the methylene blue chemical indicator was added and the bacterial colony formed was counted.

Statistical analysis. Statistical analysis was carried out via SPSS 17.0 (SPSS Inc., Chicago, IL, USA). Data were expressed as mean \pm SD. The Students' tests were used to for comparisons of HAM-D score, intestinal flora, inflammatory cytokines, NE level, BDNF level, treatment efficacy. $\mathrm{P}<0.05$ was considered to indicate a statistically significant difference.

\section{Results}

Comparison of the HAM-D score between the two groups of patients. At 3 weeks after treatment, the total HAM-D score of the two groups of patients was significantly reduced, but no statistical difference was found between them ( $p>0.05)$, suggesting that both electroacupuncture and maprotiline markedly improve the depression symptoms of the patients, and the overall efficacy of them is identical. The univariate analysis demonstrated that the improvement degrees of sleep disorders and cognitive impairment in the observation group were evidently higher than those in the control group $(\mathrm{p}<0.05)$ (Table I).

Comparison of the intestinal flora between the two groups of patients. Compared with the intestinal flora in normal individuals, the enterobacteria were increased significantly, while lactobacilli and enterococci were decreased (not shown in results) in patients with chronic diarrhea. After the treatment, the enterobacteria were reduced, while lactobacilli, enterococci and other bacteria rose again in the both groups of patients. Among them, the elevated level of lactobacilli 
Table II. Comparison of the intestinal flora between the two groups of patients (Ig CFU/g, mean \pm SD).

\begin{tabular}{|c|c|c|c|c|}
\hline \multirow[b]{2}{*}{ Intestinal flora } & \multicolumn{2}{|c|}{ Observation group $(\mathrm{n}=56)$} & \multicolumn{2}{|c|}{ Control group $(n=48)$} \\
\hline & Before treatment & After treatment & Before treatment & After treatment \\
\hline Enterobacterium & $13.94 \pm 2.83$ & $7.54 \pm 2.36$ & $14.25 \pm 3.04$ & $8.13 \pm 3.62$ \\
\hline Enterococcus & $10.68 \pm 2.37$ & $7.31 \pm 3.17$ & $11.53 \pm 3.64$ & $9.64 \pm 3.58$ \\
\hline Bacteroides fragilis & $10.78 \pm 6.35$ & $10.39 \pm 3.22$ & $11.23 \pm 7.26$ & $11.02 \pm 3.15^{\mathrm{a}}$ \\
\hline Lactobacillus & $6.67 \pm 1.36$ & $9.63 \pm 1.37$ & $6.96 \pm 1.21$ & $9.52 \pm 3.04$ \\
\hline Bifidobacterium & $9.62 \pm 0.95$ & $10.04 \pm 1.05$ & $8.43 \pm 1.03$ & $9.56 \pm 1.85$ \\
\hline
\end{tabular}

${ }^{a} \mathrm{P}<0.05$ vs. control group after treatment. $\mathrm{SD}$, standard deviation.

Table III. Comparison of the levels of NE and 5-HT before and after treatment between the two groups of patients.

\begin{tabular}{|c|c|c|c|c|}
\hline \multirow[b]{2}{*}{ Items } & \multicolumn{2}{|c|}{ Observation group $(\mathrm{n}=56)$} & \multicolumn{2}{|c|}{ Control group $(n=48)$} \\
\hline & Before treatment & After treatment & Before treatment & After treatment \\
\hline $\mathrm{NE}(\mathrm{ng} / \mathrm{ml})$ & $0.86 \pm 0.57$ & $0.59 \pm 0.23^{\mathrm{a}}$ & $0.92 \pm 0.63$ & $0.67 \pm 0.31$ \\
\hline 5-HT (ng/ml) & $79.58 \pm 43.54$ & $154.38 \pm 59.62^{a}$ & $82.43 \pm 39.84$ & $138.56 \pm 61.37$ \\
\hline
\end{tabular}

${ }^{\mathrm{a}} \mathrm{P}<0.05$ vs. control group after treatment. NE, norepinephrine; 5-HT, 5-hydroxytryptamine.
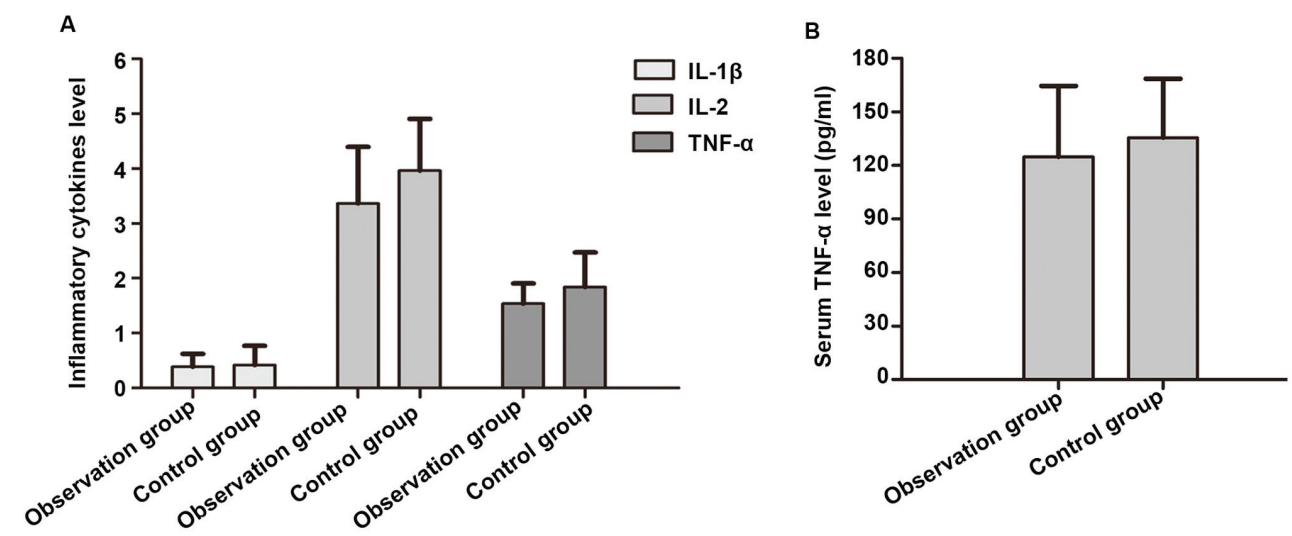

Figure 1. Comparison of the (A) serum inflammatory cytokines and (B) serum TNF- $\alpha$ after treatment between the two groups of patients. TNF- $\alpha$, tumor necrosis factor- $\alpha$; IL, interleukin.

in the observation group was obviously higher than that in the control group, with a statistically significant difference $(\mathrm{p}<0.05)($ Table II).

Effects of the cell inflammatory cytokines between the two groups of patients. Compared with those in normal patients, the serum inflammatory cytokines in the two groups before treatment were obviously increased before treatment. The levels of ILs and TNF- $\alpha$ in serum in the two groups of patients at 3 weeks after treatment returned to normal. There was no significant difference in the reduced level of inflammatory cytokines between the two groups ( $p>0.05)$ (Fig. 1A and B).

Comparison of the NE level between the two groups of patients. Compared with the normal value, before treatment, the NE levels were significantly increased, while the 5-hydroxytryptamine (5-HT) levels were significantly decreased in the two groups of patients. At 3 weeks after treatment, the NE levels were decreased to normal, and the 5-HT levels also returned to normal in the two groups of patients. Among them, the level of 5-HT in the observation group was higher than that in the control group, showing a statistically significant difference $(\mathrm{p}<0.05)$, but no significant difference in the NE level was found between the two groups (p>0.05) (Table III).

Changes in the BDNF level between the two groups of patients. Before treatment, the levels of BDNF in the two groups were significantly lower than the normal value. After treatment, the levels of BDNF in the two groups were increased, and the 
Table IV. Comparison of the efficacy of chronic diarrhea between the two groups of patients [n (\%)].

\begin{tabular}{lcccc}
\hline Groups & $\begin{array}{c}\text { Markedly } \\
\text { effective }\end{array}$ & Effective & Ineffective & $\begin{array}{c}\text { Total } \\
\text { effective rate }\end{array}$ \\
\hline Observation $(\mathrm{n}=56)$ & 32 & 21 & 3 & $94.64 \%^{\mathrm{a}}$ \\
Control $(\mathrm{n}=48)$ & 23 & 18 & 7 & $85.42 \%$ \\
\hline
\end{tabular}

${ }^{\mathrm{a}} \mathrm{P}<0.05$ vs. control group after treatment.

increased level of BDNF in the observation group was higher than that in the control group ( $<<0.05)$ (Fig. 2).

Comparison of the efficacy on chronic diarrhea between the two groups of patients. At 3 weeks after treatment, the total effective rate of the observation group was higher than that of the control group, and the difference was statistically significant $(\mathrm{p}<0.05)$ (Table IV).

\section{Discussion}

The faster pace of modern society and the increased mental stress of people has led to an increased number of mental diseases, the most common of which is depression. Depression in patients is usually manifested in the form of sleep disorders, reduced or regressed social activities, reduced desires, weight loss, hyperalgesia and autism (6). Research results have shown that the occurrence of depression in patients is associated with the excessive secretion of cytokines (7). It has been confirmed that the levels of IL-6, TNF- $\alpha$ and IL-1 are elevated in the acute phase of depression in patients, and can reflect the severity of depression and partial depression in patients (8). After patients are administered antidepressant drugs, the levels of serum IL-6 and TNF- $\alpha$ markedly decline to normal, indicating that cytokines exert certain effects in the onset of depression (9). The excessive secretion of inflammatory cytokines during the onset of depression can lead to insufficient secretion of anti-inflammatory cytokines in patients, thus causing an anti-inflammatory/inflammatory imbalance. As a chemokine, IL-8 plays a vital role in the intestinal inflammation. Secreted by intestinal epithelial cells and macrophages, IL-8 can regulate the phagocytosis of neutrophils through activation and chemotaxis. The excessive secretion of IL-8 can induce the systolic dysfunction of smooth muscle of the intestinal nervous system and influence the local intestinal neuroimmunoendocrine network system, thus triggering the intestinal mucosal inflammation (10). In addition, the excessive secretion of TNF- $\alpha$ stimulates intestinal mucosal epithelial cells, promotes the proliferation of fibroblasts and causes the degradation of intestinal mucosa epithelial function $(11,12)$. Other studies have manifested that IL-10 also exerts a vital effect in the intestinal inflammation (13). The intestinal mucosal inflammation affects the intestinal nervous system and causes abnormal smooth muscle contraction and sensitivity in the patients, thus leading to abdominal pain, abdominal distension and changes in bowel habits. Patients with these symptoms for more than 2 weeks can be diagnosed with chronic diarrhea.

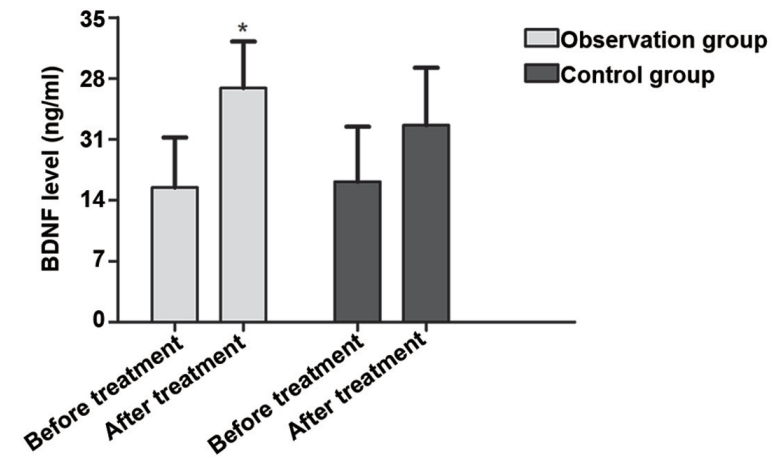

Figure 2. Comparison of the BDNF levels before and after treatment in the two groups of patients. ${ }^{*} \mathrm{P}<0.05$, compared to that before treatment. BDNF, brain-derived neurotrophic factor.

Furthermore, the occurrence of depression also has relationships to dysfunction of the central NE, 5-HT and BDNF (14-16). Research results of Karege et al (17) and Shimizu et al (18) revealed that compared with those in the normal population, the levels of BDNF and 5-HT in patients with depression are decreased, while the expression level of NE is higher than the normal value. After the drug treatment, the levels of BDNF and 5-HT in serum of patients with improved depression symptoms return to normal, but the level of NE level declines, indicating that changes in the levels of BDNF, 5-HT and NE may be associated with depression in patients $(18,19)$. The treatment for depression has achieved good results, but since drug treatment brings a variety of side effects to patients, such as xerostomia, constipation, palpitation and dizziness and the patients need to take drugs for a long time, the medication compliance is poor. Electroacupuncture treatment for depression was implemented in the 20th century with good results $(19,20)$. This study investigated the efficacy of electroacupuncture combined with probiotics for patients with depression and chronic diarrhea, so as to provide a new idea for the improvement of the patients' traditional treatment regimens.

The results of this study demonstrated that there was no significant difference in the total HAM-D score between the control group and the observation group, but the improvement degrees of sleep disorders and cognitive impairment in the former were better than that in the latter $(\mathrm{p}<0.05)$. After treatment, the cytokine levels in the two groups of patients were markedly decreased, and there was no statistical difference between the two groups ( $p>0.05$ ). The NE level in patients of the observation group was not significantly different from that in patients of the control group, but the change degrees of 5-HT and BDNF in the observation group were higher than those in 
the control group $(p<0.05)$. Moreover, the change degree of intestinal flora imbalance and the treatment effective rate in the observation group were higher than those in the control group. The above results indicate that electroacupuncture combined with probiotics is superior to traditional drug therapy in the treatment of patients with depression and chronic diarrhea, and this method is worthy of further promotion in clinical practice.

\section{Acknowledgements}

Not applicable.

\section{Funding}

The present study was funded by Guangxi Talent Highland for Zhuang and Yao Medicine and Combination of Medical Care and Elderly Care [NoTingFa(2017)44]; funded by Development Program of High-level Talent Team under Qihuang Project of Guangxi University of Chinese Medicine (grant no. 2018005); funded by Guangxi Scholarship Fund of Guangxi Education Department [No. Gui Jiao Ren (2018)18].

\section{Availability of data and materials}

The datasets used and/or analyzed during the current study are available from the corresponding author on reasonable request.

\section{Authors' contributions}

WH wrote the manuscript. WH and WG worked on treatment methods. AH and YF collected and analyzed general data of patients. YS and YC helped with scale assessment. ZT and YZ performed radioimmunoassay. WH and GF were responsible for statistical analysis. All authors read and approved the final manuscript.

\section{Ethics approval and consent to participate}

The present study was approved by the Ethics Committee of The First Clinical Faculty, Guangxi University of Traditional Chinese Medicine (Nanning, China). The signed informed consents were obtained from the patients or the guardians.

\section{Patient consent for publication}

Not applicable.

\section{Competing interests}

The authors declare that they have no competing interests.

\section{References}

1. Haraguchi A, Fukuzawa M, Iwami S, Nishimura Y, Motohashi H, Tahara Y and Shibata S: Night eating model shows time-specific depression-like behavior in the forced swimming test. Sci Rep 8: 1081, 2018.

2. Barua CC, Haloi P, Saikia B, Sulakhiya K, Pathak DC, Tamuli S, Rizavi H and Ren X: Zanthoxylum alatum abrogates lipopolysaccharide-induced depression-like behaviours in mice by modulating neuroinflammation and monoamine neurotransmitters in the hippocampus. Pharm Biol 56: 245-252, 2018.
3. Veltman EM, Lamers F, Comijs HC, Stek ML, van der Mast RC and Rhebergen D: Inflammatory markers and cortisol parameters across depressive subtypes in an older cohort. J Affect Disord 234: 54-58, 2018.

4. Innes K, Hooper J, Bramley M and DahDah P: Creation of aclinical classification. International statistical classification of diseases and related health problems--10th revision, Australian modification (ICD-10-AM). Health Inf Manag 27: 31-38, 1997.

5. Liu S and Ren JA: Pathogenesis, diagnosis and treatment of anemia in inflammatory bowel disease. Zhonghua Wei Chang Wai Ke Za Zhi 16: 398-400, 2013 (In Chinese).

6. Haack M, Hinze-Selch D, Fenzel T, Kraus T, Kühn M, Schuld A and Pollmächer T: Plasma levels of cytokines and soluble cytokine receptors in psychiatric patients upon hospital admission: Effects of confounding factors and diagnosis. J Psychiatr Res 33: 407-418, 1999.

7. Levine J, Barak Y, Chengappa KN, Rapoport A, Rebey M and Barak V: Cerebrospinal cytokine levels in patients with acute depression. Neuropsychobiology 40: 171-176, 1999.

8. Frommberger UH, Bauer J, Haselbauer P, Fräulin A, Riemann D and Berger M: Interleukin-6-(IL-6) plasma levels in depression and schizophrenia: Comparison between the acute state and after remission. Eur Arch Psychiatry Clin Neurosci 247: 228-233, 1997.

9. Słuzewska A, Rybakowski JK, Laciak M, Mackiewicz A, Sobieska $M$ and Wiktorowicz K: Interleukin-6 serum levels in depressed patients before and after treatment with fluoxetine. Ann N Y Acad Sci 762: 474-476, 1995.

10. Talati $\mathrm{M}$ and Hemnes A: Fatty acid metabolism in pulmonary arterial hypertension: Role in right ventricular dysfunction and hypertrophy. Pulm Circ 5: 269-278, 2015.

11. Du Y, Yang M, Wei W, Huynh HD, Herz J, Saghatelian A and Wan Y: Macrophage VLDL receptor promotes PAFAH secretion in mother's milk and suppresses systemic inflammation in nursing neonates. Nat Commun 3: 1008, 2012.

12. Luther J, Gala M, Patel SJ, Dave M, Borren N, Xavier RJ and Ananthakrishnan AN: Loss of response to anti-tumor necrosis factor alpha therapy in Crohn's Disease is not associated with emergence of novel inflammatory pathways. Dig Dis Sci 63: 738-745, 2018.

13. Lozovoy MA, Simão AN, Morimoto HK, Iryioda TM, Panis C, Reiche EM, Borelli SD, Oliveira SR, Cecchini R and Dichi I: Hypertension is associated with serologically active disease in patients with systemic lupus erythematosus: Role of increased Th1/Th2 ratio and oxidative stress. Scand J Rheumatol 43: 59-62, 2014.

14. Bai Y, Song L, Dai G, Xu M, Zhu L, Zhang W, Jing W and Ju W: Antidepressant effects of magnolol in a mouse model of depression induced by chronic corticosterone injection. Steroids 135: 73-78, 2018

15. Karmakar I, Haldar S, Chakraborty M, Chaudhury K, Dewanjee S and Haldar PK: Regulation of apoptosis through bcl-2/bax proteins expression and DNA damage by Zanthoxylum alatum. Pharm Biol 54: 503-508, 2016.

16. He SC, Wu S, Wang C, Du XD, Yin G, Jia Q, Zhang Y, Wang L, Soares JC and Zhang XY: Interaction between job stress and the BDNF Val66Met polymorphism affects depressive symptoms in Chinese healthcare workers. J Affect Disord 236: 157-163, 2018.

17. Karege F, Perret G, Bondolfi G, Schwald M, Bertschy G and Aubry JM: Decreased serum brain-derived neurotrophic factor levels in major depressed patients. Psychiatry Res 109: 143-148, 2002.

18. Shimizu E, Hashimoto K, Okamura N, Koike K, Komatsu N, Kumakiri C, Nakazato M, Watanabe H, Shinoda N, Okada S and Iyo M: Alterations of serum levels of brain-derived neurotrophic factor (BDNF) in depressed patients with or without antidepressants. Biol Psychiatry 54: 70-75, 2003.

19. Dong JP, Sun WY, Wang S, Wu ZQ and Liu F: Clinical observation on head point-through-point electroacupuncture for treatment of poststroke depression. Zhongguo Zhen Jiu 27: 241-244, 2007 (In Chinese).

20. Liu Q, Li B, Zhu HY, Wang YQ, Yu J and Wu GC: Glia atrophy in the hippocampus of chronic unpredictable stress-induced depression model rats is reversed by electroacupuncture treatment. J Affect Disord 128: 309-313, 2011.

This work is licensed under a Creative Commons Attribution-NonCommercial-NoDerivatives 4.0 International (CC BY-NC-ND 4.0) License. 